\title{
Recent results on diffractive and forward physics at HERA
}

\author{
Yuji Yamazaki ${ }^{1, \mathrm{a}}$ on behalf of the $\mathrm{H} 1$ and ZEUS Collaborations \\ ${ }^{1}$ Rokkodai-cho 1-1, Nada, Kobe, 657-8501 Japan
}

\begin{abstract}
.
Recent studies on diffractive processes in $e p$ scattering at HERA are reviewed. Heavy quark production in diffractive processes was studied by tagging a $D^{*}$ meson in diffractive DIS events. The cross section for events with a prompt photon in the final state was also measured. The data are well reproduced by models using diffractive parton densities determined from inclusive diffractive DIS cross sections. Two studies were also made on exclusive vector meson production. The cross-section ratio $\sigma(\psi(2 S)) / \sigma(\psi(1 S))$, which reflects the difference in transverse shape of the charmonium wave functions, are compared to various models. Moreover, the diffractive $\rho^{0}$ photoproduction with a forward neutron, which has two vertices with small- $p_{T}$ recoil, were measured to study factorisation properties. The data show some indication of re-scattering.
\end{abstract}

\section{Introduction}

HERA, the only $e^{ \pm} p$ collider, has provided opportunities to study high-energy electron-proton collisions beyond the electroweak scale. The main process, the Deep-Inelastic Scattering (DIS), probes the internal quark structure of the proton via exchanged virtual photons $\left(\gamma^{*}\right)$. The point-like nature of the virtual photon with $Q^{2} \gg \Lambda_{\mathrm{QCD}}$, where $Q^{2}$ is the negative mass of the virtual photon, ensures that the photon probes the quarkonic structure of the proton.

The DIS process can also be interpreted as collisions of $\gamma^{*} p$, which allows discussion of the DIS and photoproduction processes in the same framework; the DIS process can be considered as a special case of $\gamma^{*} p$ collisions where the $Q^{2}$ of the $\gamma^{*}$ is large ( $\left.\gg \Lambda_{\mathrm{QCD}}\right)$. In the photoproduction process, $Q^{2} \approx 0$ and no hard scale is present if there is no high- $p_{T}$ particle in the final state and the process is described by the exchange of particles. In processes where a hard scale is present, individual partons are visible and the cross sections can be calculated in perturbative QCD.

Diffractive processes at HERA are characterised as a colourless exchange between the virtual photon and the proton, where the photon system dissociates into a hadronic system $X$ while the proton may remain intact or may dissociate to a system $Y$. The the momentum exchanged at the $p \rightarrow Y$ vertex, $t$, is typically small ( $-t \leq \Lambda_{\mathrm{QCD}}$ ) unless the mass of the system $Y$ is large. There it is expected that the exchanged state at the proton vertex is particle-like e.g. pions, Reggeons or "Pomerons" (IP). It may be, however, that the exchange can be explained by partonic colourless states e.g. (dressed) two gluons and can be interpreted by pQCD calculations.

\footnotetext{
ae-mail: yamazaki@phys.sci.kobe-u.ac.jp
} 

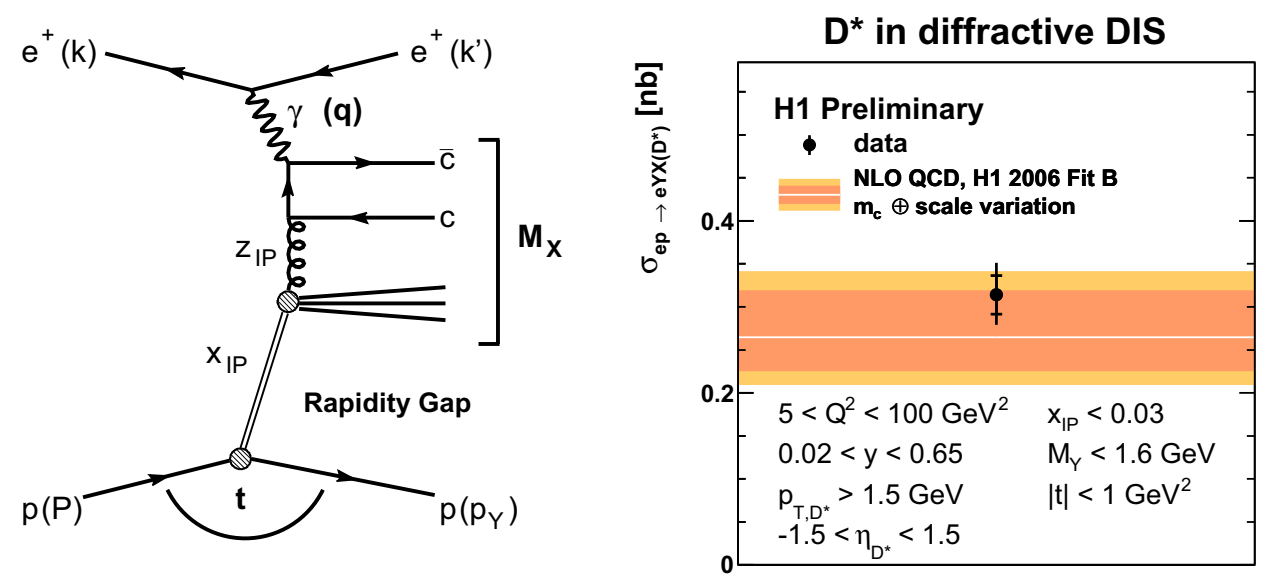

Figure 1. (left) A typical diagram of charm quark pair production in photon-dissociative diffractive processes in $e p$ scattering. (right) The charm quark production cross section in comparison to the NLO QCD calculation using H1 2006 Fit B diffractive PDF [1].

HERA allows quantitative investigations to be made of the partonic content of such exchanges by measuring the cross section behaviour of Diffractive DIS (DDIS), which is defined as DIS processes with diffractive final states (experimentally defined as a presence of large rapidity gap, observation of the fast-going proton or neutron, etc.). The gluon density in the exchange can also be extracted through the scaling violation behaviour of the DDIS cross sections in $Q^{2}$ (for example in Ref. [1]), provided that the factorisation holds between the $\gamma^{*}$ vertex and other part of the DDIS diagram. The factorisation was theoretically proven for DDIS but not for other processes, such as diffraction in photoproduction or hadron-hadron collisions, even with a presence of a hard scale. The factorisation breaking may lead to "absorption" or re-scattering between two diffractive systems. The cross sections are then suppressed from the theoretical predictions based on the diffractive parton densities extracted from DDIS. Such behaviour was first largely advertised by the Tevatron measurements on dijet production in diffraction [2]. There are several observations also at HERA, e.g. photoproduced dijets [3] as well as the $t$-distribution in forward neutron production [4].

In the following sections, four recent results are presented. $D^{*}$ production in diffractive DIS [5] and prompt photon production in diffractive photoproduction [6] are compared to the models based on the diffractive PDFs. The production ratio $\psi(2 S) / \psi(1 S)$ in diffractive DIS [7] is compared to various models with approaches based on perturbative QCD in Section 4. Exclusive production of $\rho^{0}$ in photoproduction with a fast neutron [8] is reviewed in Section 5 in the context of the absorptive phenomena. 

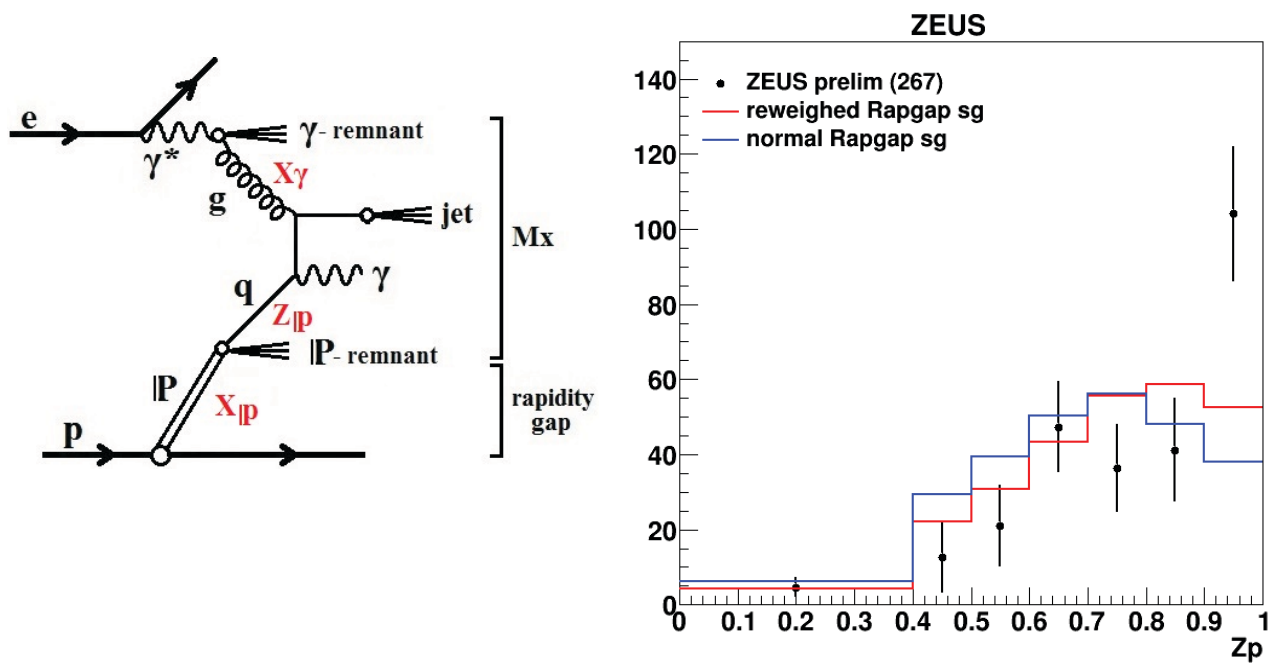

Figure 2. (left) An example of diagrams where a prompt photon is produced with a quark jet in the resolved diffractive photoproduction process in $e p$ scattering. (right) The $z_{\mathbf{p}}$ dependence of the cross sections with a prompt photon, a jet and a large rapidity gap in photoproduction.

\section{$2 D^{*}$ production in diffractive DIS}

Charm quarks in diffractive DIS are predominantly produced through the so-called boson-gluon fusion diagram (Fig. 1(left)). The cross sections with a $D^{*}$ in diffractive DIS are therefore sensitive to the gluonic content of the diffractive exchange.

The cross section was measured with $281 \mathrm{pb}^{-1}$ of $e p$ collisions by the $\mathrm{H} 1$ Collaboration in the deep-inelastic regime $\left(5<Q^{2}<100 \mathrm{GeV}^{2}\right)$ with a presence of a large rapidity gap from the edge of the detector in the proton direction, hence selecting either events with proton staying intact or dissociated to a small mass state $Y$.

The cross section (Fig. 1(right)) shows good agreement with the NLO prediction within the uncertainty if using the diffractive PDF H1 2006 Fit B [1] extracted from the scaling violation of the inclusive diffractive DIS data. This indicates that the factorisation holds in diffractive DIS. Differential cross sections are also measured as a function of other variables described in Fig. 1(right). All the distributions are well reproduced by the NLO calculation, providing a cross-check on the diffractive parton densities, in particular the gluon density.

\section{Prompt photon production in diffractive photoproduction}

This measurement requires an isolated photon and a jet in the photon dissociation system in addition to the presence of a large rapidity gap in photoproduction events. The advantage of a photon + a jet over dijet events is (a) less sensitive to the QCD radiation and (b) more sensitivity on quark content of the diffractive exchange as the prompt photon couples only to quarks (Fig. 2(left)).

The fraction of parton momentum involved in the hard scattering with respect to the two incoming "particles", for the photon $\left(x_{\gamma}\right)$ and for the diffractive exchange $\left(z_{\mathbb{P}}\right)$, can be reconstructed using the momentum of the photon and jet. The events are further classified as "direct" and "resolved" events using the reconstructed $x_{\gamma}$. In this analysis, $x_{\gamma}>0.8$ is defined as direct-enriched region and the rest 


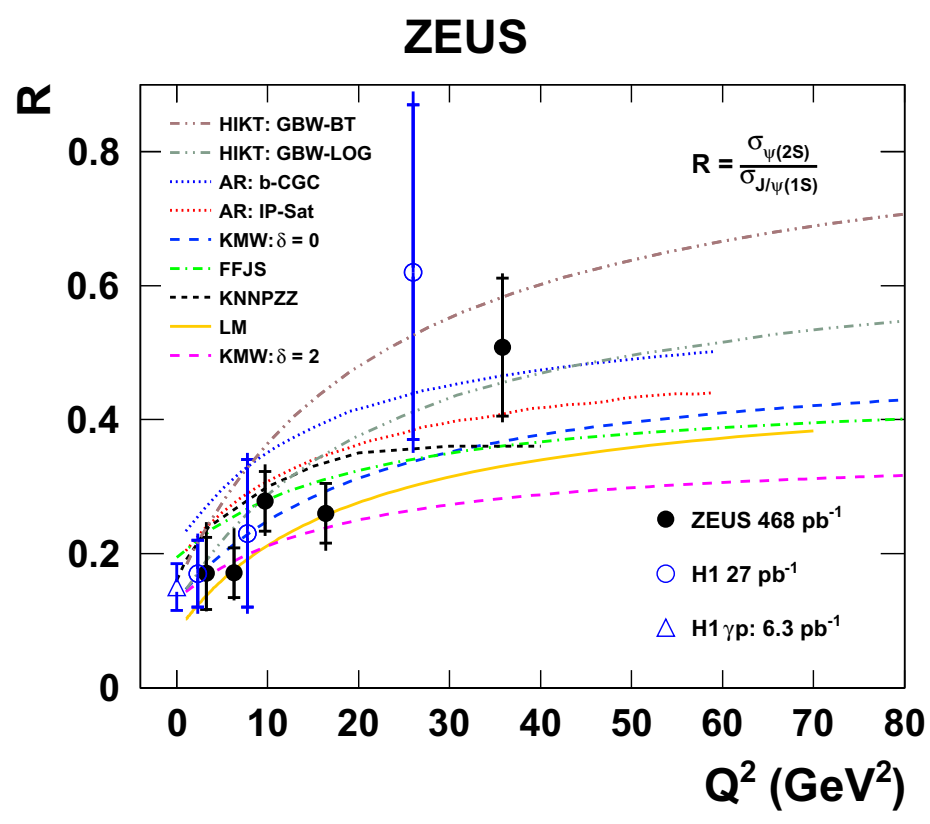

Figure 3. The cross section ratio $\sigma(\psi(2 S)) / \sigma(\psi(1 S))$ as a function of $Q^{2}$ in comparison to various models.

is resolved-enriched. The fraction of the resolved and direct processes in the RAPGAP model was reweighted so as to describe the data.

Theoretical prediction by RAPGAP reproduces well various distributions, such as $E_{T}$ and $\eta$ of the photon and jets. The only exception is $z_{\mathbf{P}}$ distribution (Fig. 2(right)). It fails to describe the peak near $z_{\mathbf{P}}=1$ observed in the data. This may indicate that the model lacks some diagrams like two partons in the diffractive exchange with very asymmetric configuration i.e. one of the two partons carries almost the entire momentum of the diffractive exchange. Such diagrams are higher-twist contribution and not included in the framework of the QCD fit to diffractive DIS cross sections.

\section{The production ratio $\psi(2 S) / \psi(1 S)$ in diffractive DIS}

This process measures the cross section ratio of the quasi-elastic charmonium production $e p \rightarrow e \psi Y$ where $Y$ is either a proton or a state with small mass. The ratio $\sigma(\psi(2 S)) / \sigma(\psi(1 S))$ is expected to have a strong dependence in $Q^{2}$, which is originated from different wave function shape of $1 S$ and $2 S$ onium states. The wave function of the $1 S$ state is always positive, while the function for the $2 S$ state has a node at around $0.35 \mathrm{fm}$.

The vector boson is generated from a virtual photon dissociated into a $q \bar{q}$ state. The transverse profile of the $q \bar{q}$ state wave function is similar to that of the charmonium for low $Q^{2}\left(Q^{2} \simeq m_{\psi}^{2}\right)$. In this case, the convolution of the two wave functions, $q \bar{q}$ state and the $\psi(2 S)$ state, may become small since the wave function changes the sign along the transverse position. The $q \bar{q}$ state, however, becomes much smaller than the $\psi$ wave function for $Q^{2} \gg m_{\psi}$ and it becomes likely that the two quarks probe similar values of wave functions. No such cancellation is expected in the convolution in this case. This predicts that the cross section ratio is to increase with $Q^{2}$. 


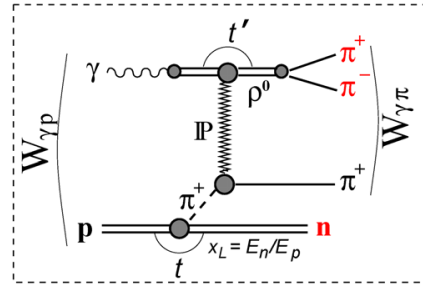

(a)

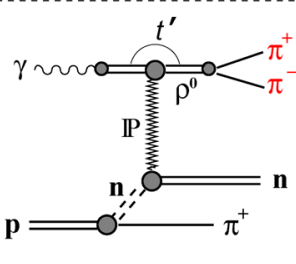

(b)

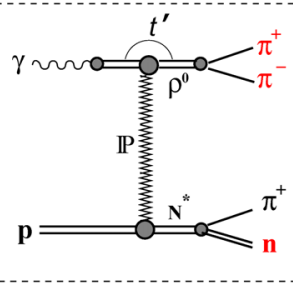

(c)



(d)

Figure 4. Diagrams for processes contributing to $\rho^{0}$ production with a leading neutron at HERA. Three processes are considered as signal: (a) pion exchange (b) neutron exchange and (c) a neutron produced from the decay an excited nucleon or from a non-resonant $\pi+n$ state. The event with a neutron produced from dissociation of the system $Y$ is considered as background (d).

The data in Fig. 3 was measured with full luminosity of HERA runs and greatly improves the precision of the previous measurement. The data confirms qualitatively the above-explained expectation. Looking into detail, the data behaviour is consistent with many of the models although some of the models are excluded. The ratio is flat against other variables like $W$, the centre-of-mass energy of the $\gamma^{*} p$ system, and $|t|$.

\section{Exclusive $\rho^{0}$ photoproduction with a fast neutron}

Exclusive $\rho^{0}$ production with a fast neutron is a process without the explicit presence of a hard scale since both the outgoing neutron and $\rho^{0}$ do not carry high transverse momentum. It is therefore appropriate to describe the process in terms of particle exchange. For events with large $x_{L}=E_{n} / E_{p}$, the dominant process is one-pion exchange (OPE) (Fig. 4 (a)). This allows the diffractive production of $\gamma \pi$ scattering to be studied, especially by comparing to the results from exclusive production of $\rho^{0}$ without a tagged neutron.

There are other two other diagrams (Fig. 4 (b) and (c)), which do not leave any significant activity in the forward part of the detector and are considered as signal in this analysis. The neutrons through the proton dissociation system $Y$ is considered as background and was subtracted.

The shape of the measured neutron spectrum is well reproduced by choosing appropriate "pion flux" models among those proposed (see the Ref.[8] for more detail). The production rate is, however, suppressed by about a factor two. The cross section ratio $\sigma_{\gamma \pi \rightarrow \rho^{0} \pi} / \sigma_{\gamma p \rightarrow \rho^{0} p}$ was measured $0.25 \pm 0.06$, which is far less than that expected from the additive quark model $(\sim 0.6)$. Similar suppression was observed in inclusive neutron production[10]. This may be an indication of absorptive correction.

The exponential slope parameters of the leading neutron $b_{n}$ determined from the $p_{T}$ dependence $\sigma\left(x_{L}, p_{T}\right)=A \exp \left(b_{n} p_{T}^{2}\right)$ was measured at HERA for inclusive neutron production [9] and compared to models with various pion flux, giving constraint to models (Fig. 5(right)). A similar measurement was made for the exclusive $\rho^{0}$ production and is shown in Fig. 5. The behaviour is quite different from the inclusive measurement at high $x_{L}$. This may be a further indication of the presence of re-scattering, which predicts to suppress the events with a neutron with large $p_{T}$.

\section{References}

[1] H1 Collaboration, A. Aktas et al., Eur. Phys. J. C48, 715 (2006). 

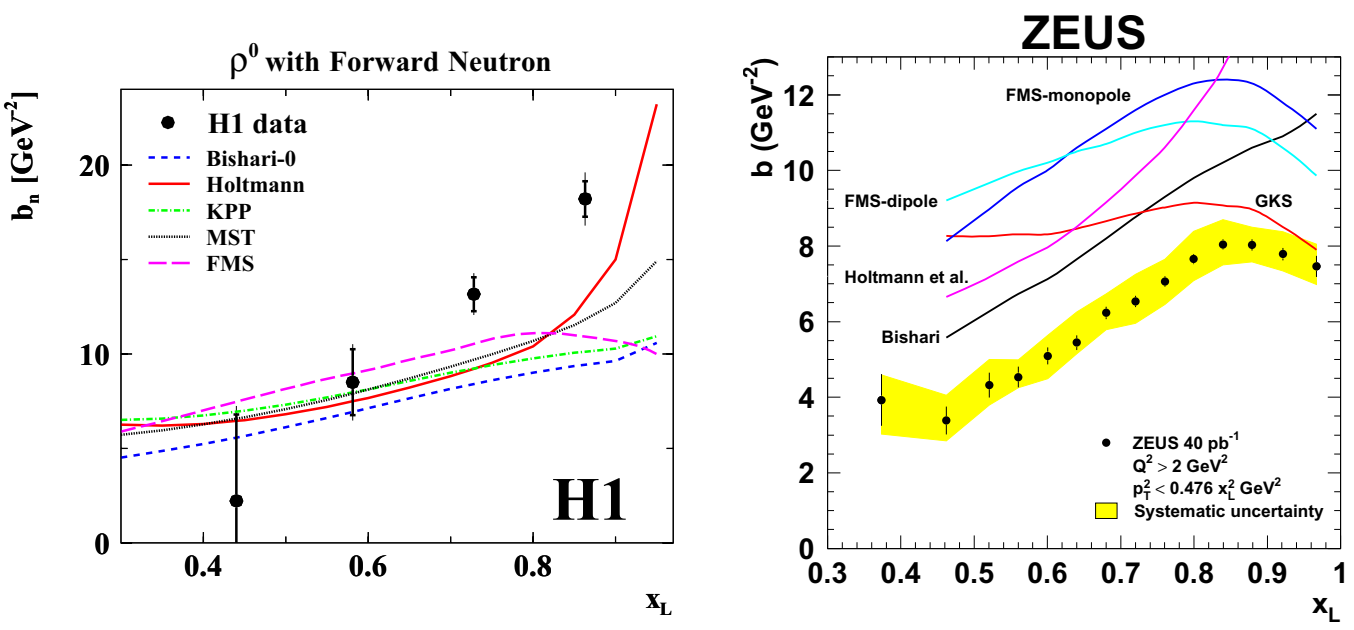

Figure 5. (left) The exponential slope parameter $b_{n}$ for the exclusive $\rho^{0}$ production. (right) The corresponding parameters for inclusive DIS with a leading neutron[9].

[2] CDF Collaboration, T. Affolder et al., Phys. Rev. Lett. 84, 5043 (2000).

[3] H1 Collaboration, A. Aktas et al., Eur. Phys. J. C51, 549 (2007).

[4] ZEUS Collaboration, S. Chekanov et al., Nucl. Phys. B776, 1 (2007).

[5] H1 Collaboration, H1prelim-16-011, available under:

http: //www-h1.desy.de/h1/www/publications/htmlsplit/H1prelim-16-011. long.html.

[6] ZEUS Collaboration, ZEUS-prel-15-001, available under:

https://inspirehep.net/record/1368789? ln=en

[7] ZEUS Collaboration, H. Abramowicz et al., Nucl. Phys. B909, 934 (2016).

[8] H1 Collaboration, V. Andreev et al., Eur. Phys. J. C76, No. 1, 41 (2016).

[9] ZEUS Collaboration, S. Chekanov et al, Nucl. Phys. B776, 1 (2007).

[10] ZEUS Collaboration, S. Chekanov et al, Nucl. Phys. B637, 3 (2002); H1 Collaboration,

F.D. Aaron et Al, Eur. Phys. J C68, 381 (2010). 